\title{
Acquired Haemophilia A. Which is the best therapeutic choice in older adults? Single center study of 4 cases
}

\author{
E. Mauro, E. Garlatti Costa, A. Zanier, M. Maset, A. Ermacora, M. Ghersetti, P. Casarin \\ Department of Internal Medicine, Pordenone General Hospital, Italy
}

\begin{abstract}
SUMMARY
Acquired haemophilia A (AHA) is a rare bleeding disorder due to autoantibodies directed against coagulation factor VIII. The treatment is based on recombinant activated factor VII and activated prothrombin complex concentrate. However, mainly in older patients, severe thrombotic complications have been reported. Here we report the different therapeutic approaches in 4 cases of elderly patients with AHA and co-morbidities.
\end{abstract}

Key words: Acquired haemophilia A; Recombinant activated factor VII; Activated prothrombin complex concentrate.

Reumatismo, 2019; 71 (1): 37-41

\section{INTRODUCTION}

cquired haemophilia A (AHA) is a bleeding disorder due to autoantibodies directed against coagulation factor VIII (1). AHA is rare with an estimated incidence of 1.5 cases per million per year (2). The median age seems to be 78 years and increases with age (2). The treatment is based on haemostatic and immune-modulating agents (3).

In particular, from congenital haemophilia experience, bypassing agents, i.e. recombinant activated factor VII (rFVIIa) and activated prothrombin complex concentrate (aPCC) are considered the first choice of treatment in combination with immunemodulating agents in $\mathrm{AHA}(4,5)$.

However, in the bypassing agents treatment, severe thrombotic complications have been reported $(6,7)$.

Therefore, in treatment of AHA, age of patient, underlying diseases and related therapy have to be carefully evaluated. In the present paper, we retrospectively evaluated the clinical and therapeutic features of 4 AHA patients treated at our Institution.

\section{CASE REPORTS}

\section{Case 1}

A 71-year old male was referred to our Medical Department with recent-onset hemarthrosis of the right knee. His medical history reported paroxysmal atrial fibrillation in oral anticoagulant therapy (Warfarin), hypertensive cardiomyopathy with congenital atrial septal defect and chronic obstructive pulmonary disease. Clinical and laboratory data are summarized in Table I. Two packed red blood cells were infused, knee arthrocentesis confirmed hemarthrosis, while a fluorodeoxyglucose positron emission tomography was negative for other bleeding sites, malignancies or autoimmune diseases. Anticoagulant therapy was stopped, infusion of vitamin $\mathrm{K}$ and one unit of fresh frozen plasma were started. However, plasma infusion was stopped because of orticarioid reaction, angioedema and dyspnoea. Considering transfusion reaction and the clinical hemodynamic stability, the AHA was managed only with immunosuppressive therapy (prednisone 1 $\mathrm{mg} / \mathrm{kg}$ and cyclophosphamide $1.4 \mathrm{mg} / \mathrm{kg}$ ), without prescribing bypassing agents. Co-
Corresponding author: Endri Mauro Department of Internal Medicine, Pordenone General Hospital Via Montereale, 24 - Pordenone, Italy E-mail: endri76@libero.it 
agulation and FVIII levels returned to normal range within 20 days. Immunosuppressive treatment was well tolerated and was continued for two months until eradication of the FVIII inhibitor.

\section{Case 2}

An 84-year old male was admitted because of spontaneous cutaneous bleeding, retropharyngeal hematoma and abdominal pain spreading to the lower left leg. In medical history, arterial hypertension has been reported and anti-platelet therapy (acetylsalicylic acid) due to intraseptal atrial aneurism was ongoing. Clinical and laboratory data are summarized in Table I; an autoimmunity panel test was negative. Total body computed tomography (CT) scan revealed a large hematoma of the left iliopsoas muscle $(9 \times 9 \mathrm{~cm})$. Anti-platelets therapy was discontinued and two units of packed red blood cells were infused. Treatment with rFVIIa (recombinant activated factor VII, Novoseven ${ }^{\circledR}$ ) was started at a dose of 90 $\mu \mathrm{g} / \mathrm{kg}$ every 6 hours for 6 days, then every 8 hours for 1 day, then every 12 hours for 4 days and finally once daily for 2 days. Immunosuppressive therapy with prednisone $1.3 \mathrm{mg} / \mathrm{kg}$ and cyclophosphamide $100 \mathrm{mg} /$ daily $(1.3 \mathrm{mg} / \mathrm{kg})$ was started. Coagulation and FVIII levels went back into normal range within 24 days. Eradication of the FVIII inhibitor was achieved after 4 weeks of treatment. In the follow up, no thrombotic events were reported and a control abdominal CT scan confirmed progressive reduction of the iliopsoas hematoma.

\section{Case 3}

A 69-year old male was admitted to our Institution because of extensive skin ecchymoses of the upper and lower limbs, mandibular hematoma of the neck and arthralgias. His medical history showed diabetes mellitus and anti-platelet therapy due to pericarditis. Laboratory and clinical data are summarized in Table I; an autoimmunity test panel was negative. CT scan was negative for deep muscular or retroperitoneal hematomas or occult malignancies. The patient was transfused with 4 packed red blood cells. Antiplatelet therapy was promptly

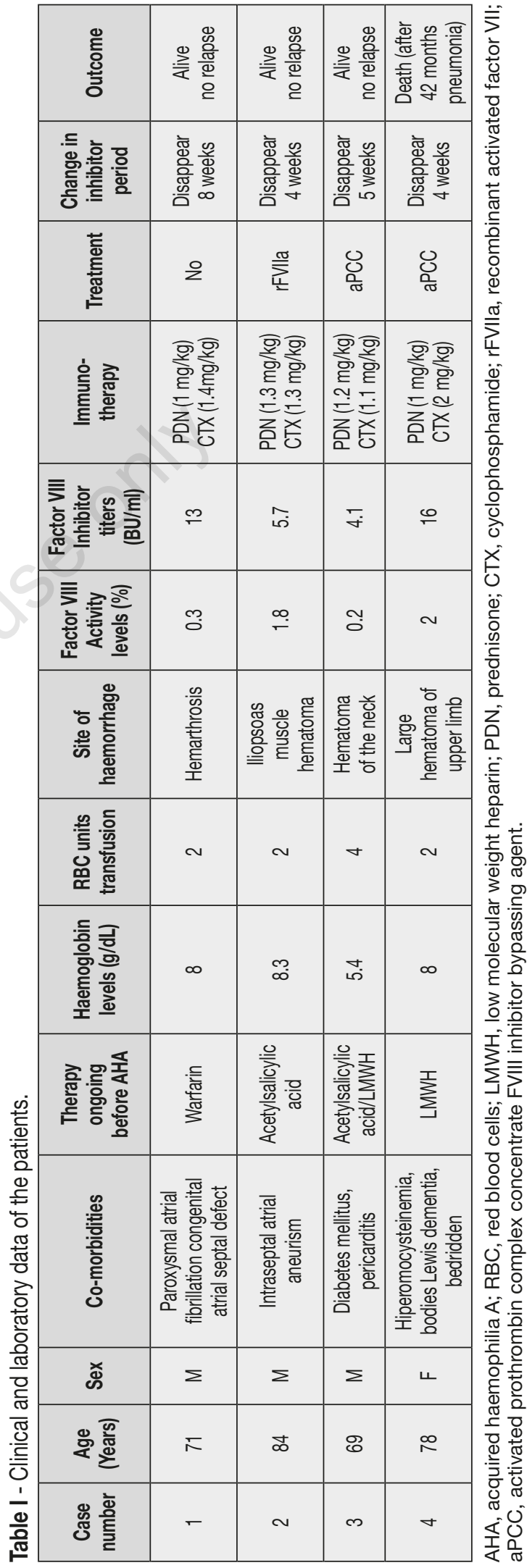


discontinued. Treatment with activated prothrombin complex concentrate (aPCC) $\left(\right.$ FEIBA $\left.^{\circledR}\right)$ was started at a dose of $70 \mathrm{U} /$ $\mathrm{kg}$ every 12 hours for 4 days, then $66 \mathrm{U} / \mathrm{kg}$ every other day for the following 9 days, then $44 \mathrm{U} / \mathrm{kg}$ every other day for 18 days in combination with immunosuppressive therapy (6-metilprednisolone $1.2 \mathrm{mg} / \mathrm{kg}$ and cyclophosphamide $100 \mathrm{mg}$ daily, $1.1 \mathrm{mg} / \mathrm{kg}$ daily). Bypassing agent was stopped after 5 weeks and immunosuppressive therapy after 40 days respectively. Eradication of the FVIII inhibitor was achieved after 30 days of treatment. No thrombotic events were reported during and after this treatment.

\section{Case 4}

A 78 old female was admitted to our Institute because of a large hematoma of upper right limb. Her medical history showed: hyperhomocysteinemia, chronic obstructive pulmonary disease, hepatitis $\mathrm{C}$ virus infection without signs of chronic liver disease, Lewy body dementia. Moreover, the patient was bedridden and a thromboprophylaxis with low molecular weight heparin (LMWH) was ongoing. Clinical and laboratory data are summarized in Table I. Ultrasound evaluation of upper right limb reported a large edema and hematoma among muscles planes, total body CT scan was negative for occult malignancies. LMWH was promptly stopped and treatment with activated prothrombin complex concentrate (aPCC) $\left(\right.$ FEIBA $\left.^{\circledR}\right)$ was started at a dose of $100 \mathrm{U} / \mathrm{kg}$ every 6 hours for 5 days in combination with prednisone $1 \mathrm{mg} /$ $\mathrm{kg}$ daily and cyclophosphamide $2 \mathrm{mg} / \mathrm{kg}$ daily. The patient underwent 2 packed red blood cell transfusions. Progressive clinical remission of hematoma was achieved in 3 weeks. According to clinical improvement, after 4 weeks, activated partial thromboplastin time and FVIII activity went back into normal range and FVIII inhibitor was undetectable. Immunosuppressive therapy was stopped after 6 weeks.

\section{DISCUSSION}

AHA is a rare bleeding disorder, therefore knowledge about well-defined treatment schedules and real evaluation of therapyrelated adverse events (AEs) therapy related are lacking. Treatment is a combination of immunosuppressive therapy and pro-coagulant factors; in particular the activated prothrombin complex concentrate (aPCC) FVIII inhibitor bypassing activity or recombinant activated factor VII (rFVIIa) $(4,5,8)$. The use of bypassing agents in AHA has been directly translated from congenital haemophilia A (5). However, not only in acquired, but also in congenital haemophilia, an increased incidence of thromboembolic therapy-related events has been reported. Indeed, a review by Aledort of the Food and Drug Administration's Med Watch pharmacovigilance program database from 1999 through 2002 showed an incidence of thromboembolic events of 8.24 per 100,000 aPCC $\left(\right.$ FEIBA $\left.^{\circledR}\right)$ infusions (9). Herlich and co-workers reported an incidence of total thrombotic adverse events for FEIBA $^{\circledR}$ of 4.05 per 100,000 infusions for the period 1990 through 1999; in particular 16 thrombotic AEs have been recorded of which 3 cases in AHA with risk factors (obesity, coronary artery disease, but also drug overdose) (10). Such factors may be also involved in rFVIIa related thrombotic AEs (11). Treatment with either rFVIIa or aPCC is characterized by a higher incidence of arterial and venous thrombosis in AHA than in congenital haemophilia, probably due to related clinical risk factors (such as age and co-morbidities) (4). More recently, with regard to thrombotic therapy related events in AHA, Sumner and co-workers showed a mainly arterial thrombosis incidence of $8.6 \%$ in 139 cases treated with rFVIIa, while EACH2 study recorded $2.7 \%$ of thrombotic events due to rFVIIa and $3.6 \%$ due to aPCC respectively (6). On the other hand, in the two studies with the largest cohort of patients with AHA $(4,7)$, more than $80 \%$ of them were older adults ( $>65$ years old). Moreover, in real practice, the medical history of patients with AHA is characterized by previous diseases at thromboembolic risk such as atrial fibrillation, deep vein thrombosis, pulmonary embolism, diabetes mellitus, obesity, hypercholesterolemia, 
cancers $(12,13)$, and antiplatelet drugs and/or anticoagulant therapy. However in the European Acquired Haemophilia study (7) and in the United Kingdom Haemophilia Centre Doctors' Organisation study (4), co-morbidities and antiplatelet/anticoagulant drugs intake are not recorded. In 2013, the outcome of acquired haemophilia $\mathrm{A}$ in France from the prospective SACHA (Survelliance des Auto anti Corps au cours de l'Hémophilie Acquise) study was reported (12). In this study, 82 consecutive patients were enrolled, with median age of 76.7 years (range 25-103); 28 cases were treated with rFVIIa alone and 6 cases with aPCC alone. This study confirmed the higher prevalence of AHA in the older adult population. No thromboembolic events were reported. More recently, the first prospective multicentre study on Factor VIII inhibitor bypass activity $\left(\right.$ FEIBA $\left.^{\circledR}\right)$ was published (13). In this study, 34 consecutive patients were included (aged from 59 to 97 years): 5 FEIBA $^{\circledR}$ related AEs were recorded. All AEs were thrombosis related or indicative of a potentially hypercoagulable state. In our series of patients, at onset, we evaluated the thromboembolic risk. In particular, in case 1 , the medical history showed atrial fibrillation and congenital atrial septal defect in oral anticoagulant therapy, in case 2 intraseptal atrial aneurism in antiplatelet therapy, in case 3 diabetes mellitus in acetylsalicylic acid treatment while case 4 was bedridden with hyperhomocysteinemia and in LMWH prophylaxis. All patients needed packed red cells transfusions. Concerning onset of AHA, case 1 showed a hemarthrosis of the right knee, case 2 was admitted because of retropharyngeal hematoma and a large hematoma of the left iliopsoas muscle, cases 3 and 4 were characterized by a submandibular hematoma of the neck and by a arge hematoma of the upper right limb, respectively. Therefore, at onset, the bleeding risk was not only due to AHA per se but also to anticoagulant/antiplatelet drug intake while the mandatory discontinuation of antithrombotic therapy, the underlying vascular diseases and the age put the patient at high thromboembolic risk. All these together highlight the com- plex management of AHA in older adults with cardiovascular co-morbidities. In literature, studies with small series of older patients have evaluated the safety and efficacy of modified schedules therapy with procoagulant agents. In particular, Zanon and co-workers showed the efficacy and the safety of a high dose of human plasmaderived FVIII-von Willebrand Factor in patients with AHA and concomitant cardiovascular disease during antiplatelet drug treatment (14). On the other hand, Makoto and Di Capua reported a series of 7 and 4 cases, respectively, where AHA remission had been achieved by low-dose recombinant rFVIIa low dose or by immunosuppressive therapy alone $(8,15)$. In particular, Makoto and co-workers showed AHA remission with immunosuppressive therapy alone in one patient with major bleeding. Even in our series of cases, patient number 1, with hemarthrosis, considered as major bleeding, achieved AHA remission by immunosuppressive therapy alone. Therefore the corner stone of AHA therapy seems to be the fast inhibitor removal, likely to be effective even in selected cases with severe haemorrhage. To the best of our knowledge, we are reporting the second AHA case with major bleeding treated with immunosuppressive therapy alone. Although the cases are few, we have highlighted the efficacy and the safety of aPCC (FEIBA $\left.{ }^{\circledR}\right)$ and recombinant activated FVII (rFVIIa) standard dose in older adults with cardiovascular diseases in antithrombotic therapy. In conclusion, recognition of thrombotic risk factors and attention to drug dose play a key role in avoiding thrombotic AEs in particular settings of older adult patients with co-morbidities and multi-drug intake. However, considering the different experiences in the field of AHA treatment, prospective studies involving a large number of patients are required.

\section{REFERENCES}

1. Green D, Lechner K. A survey of 215 non hemophilic patients with inhibitors to Factor VIII. Thromb Haemost. 1981; 45: 200-3.

2. Collins WP, Hirsch S, Baglin TP, et al. Acquired hemophilia $\mathrm{A}$ in the United Kingdom: 
a 2 year national surveillance study by the united Kingdom Haemophilia Center Doctors' Organisation. Blood. 2007; 109: 1870-7.

3. Kessler CM, Nemes L. Acquired inhibitors to factor VIII. In: Rodriguez EC, Lee CA, editors. Inhibitors in patients with haemophilia. Blackwell Publishing. 2002; 98-112.

4. Collins WP, Chalmers E, Hart D, et al. Diagnosis and management of acquired coagulation inhibitors: a guideline from UKHCDO. Br J Haematol. 2013; 162: 758-73.

5. Huth-Kühne A, Baudo F, Collins P, et al. International recommendations on the diagnosis and treatment of patients with acquired hemophilia A. Haematologica. 2009; 94: 566-75.

6. Sumner MJ, Geldziler BD, Pedersen M, Seremetis S. Treatment of acquired haemophilia with recombinant activated FVII: a critical appraisal. Haemophilia. 2007; 13: 451-61.

7. Baudo F, Collins P, Huth-Kühne A, et al. Management of bleeding in acquired hemophilia A: results from the European Acquired Haemophilia (EACH2) Registry. Blood. 2012; 120: 39-46.

8. Makoto S, Kanaya M, Izumiyama K, et al. Treatment of bleeding in acquired hemophilia A with the proper administration of recombinant activated factor VII: single center study 7 cases. Int J Gener Med. 2016; 9: 393-9.

9. Aledort LM. Comparative thrombotic event incidence after infusion of recombinant factor VIIa versus factor VIII inhibitor bypass activity. J Thromb Haemost. 2004; 2: 1700-8.
10. Ehrlich HJ, Henzi MJ, Gomperts ED. Safety of factor VIII inhibitor bypass activity (FEIBA): 10 years compilation of thrombotic adverse events. Haemophilia. 2002; 8: 83-90.

11. Peerlinck K, Vermylen J. acute myocardial infarction following administration of recombinant activated factor VII (NovoSeven) in a patients with haemophilia A and inhibitor. Thromb Haemost. 1999; 82: 1775-6.

12. Borg JY, Guillet B, Le Cam-Duchez V, et al. outcome of acquired haemophilia in France: the prospective SACHA (Survelliance des Auto antiCorps au cours de l'Hémophilie Acquise) registry. Haemophilia. 2013; 19: 564-70.

13. Borg YJ, Négrier C, Duriueu I, et al. FEIBA in the treatment of acquired hemophilia A: results from the prospective multi center French "FEIBA dans l'hémophilie A acquise (FEIBHAC) registry. Haemophilia. 2015; 21: 330-7.

14. Zanon E, Milan M, Brandolin B, et al. High dose of human plasma derived FVIII-VWF as first line therapy in patients affected by acquired hameophilia A and concomitant cardiovascular disease: four case report and literature review. Haemophilia. 2013; 19: e48-e61.

15. Di Capua M, Coppola A, Nardo A, et al. Management of bleeding in acquired haemophilia A with recombinant activated factor VII: does one size fit all? A report of four cases. Blood Tranfus. 2015; 13: 328-32. 